\title{
Vibration of Axially Strained Triple-Wall Carbon Nanotubes
}

\author{
C. Y. Wang*, Y. Zhao, S. Adhikari, and Y. T. Feng \\ School of Engineering, Swansea University, Singleton Park, Swansea, Wales SA2 8PP, UK
}

\begin{abstract}
A comprehensive study is conducted on the vibration of axially strained triple-wall carbon nanotubes (TWCNTs). The Flugge shell theory is used, which largely improves the accuracy and enhances the scope of the research. It is found that the tensile or compressive axial strain can strengthen or weaken the structural rigidity of a TWCNT and thus, substantially up- or down-shift its fundamental frequency. The strongest effect occurs for the lowest frequency associated with bending mode with a circular cross-section and radial modes with two to three circumferential waves depending on the radius and axial wavelength of TWCNTs. In addition, torsional, longitudinal or intertube radial modes largely controlled by the material constants or the interlayer van der Waals interaction coefficient are insensitive to the initial axial strain.
\end{abstract}

Keywords: Carbon Nanotubes, Triple Walls, Vibration

\section{INTRODUCTION}

The discovery of carbon nanotubes $(\mathrm{CNTs})^{1}$ has excited a significant wave of research on their unique mechanical behaviors ${ }^{2,3}$ and potential applications in nanotechnology. ${ }^{4,5}$ It is found that, due to their extreme elastic modulus and low mass density, CNTs can serve as nanoscale resonators whose frequency is up to the order of magnitude of terahertz. ${ }^{6-8}$ This suggests that CNTs are promising for nanoscale transducers, actuators and chemical/mechanical sensors whose performance depends crucially on the dynamical response of CNTs. Thus, the vibration and wave propagation properties of CNTs become a major issue of great interest in nanomechanics. ${ }^{6-12}$

In the application of CNTs, they are subjected to various mechanical loadings, e.g., axial force and radial pressure, which can arise from thermal stress, lattice mismatch of different materials, applied electrical voltage or initial external load. These initial loadings could exert strong influence on the dynamical behavior of CNTs and in turn, significantly affect the proper functioning of CNT-based nanostructures. On the other hand, the dependence of the vibration frequency and the wave propagation speed of CNTs on the initial loadings is potentially useful for many CNT-based nanodevices. A typical example is the CNT strain/pressure sensors ${ }^{13-15}$ whose principle of sensing is based on the relationship between the initial stain and resulting frequency shift. Moreover, since the delamination

*Author to whom correspondence should be addressed. in composites would alter the local strain/stress field in matrix, such CNT strain sensors ${ }^{13-15}$ have the potential to be utilized for the health monitoring of composites. Thus, the effect of initial loadings on the dynamical behavior of CNTs has attracted considerable attention in recent research. $^{13,15-20}$ In 2004, using an atomistic model, Li and $\mathrm{Chou}^{12}$ first studied the lowest frequency vibration of single-wall CNTs (SWCNTs) under axial force or radial pressure. Beam models were employed by Zhang et al. ${ }^{16}$ and Cai and Wang ${ }^{17,18}$ to investigate the effect of an initial axial load on the transverse modes of SWCNTs and multiwall CNTs (MWCNTs). These studies ${ }^{13,16-18}$ show that the frequency up-shifts with tensile loads, but decreases with compressive loads. In 2007, Sun and Wang ${ }^{19}$ further examined this issue for the radial vibration using a thin shell model based on the single Donnell equation. ${ }^{9}$ They found that only the fundamental frequency is sensitive to the axial loading. It has been noted that the single Donnell equation is unable to account for the torsional and longitudinal vibration ${ }^{11}$ and it is not applicable for slender MWCNTs with a large length-to-radius ratio. ${ }^{9}$ Very recently, more accurate Flugge shell equations, ${ }^{9}$ previously used for free vibration of CNTs, ${ }^{11,12}$ were employed by Selim et al. ${ }^{20}$ to study the wave propagation of axially compressed MWCNTs. ${ }^{18}$ It was shown that the propagation speed can be raised by applying the tensile force.

Motivated by these earlier studies that mainly focused on some particular cases, the present work aims to understand the general vibration behavior of the axially 
strained triple-wall CNTs (TWCNTs). The major attention is focused on the effect of the axial strain on all possible vibration modes of TWCNTs. The dependence of such an effect on the radius and axial wavelengths of TWCNTs will also be examined in details. In addition, efforts will be made to provide physical explanation of the numerical results. To this end, the two dimensional shell model ${ }^{24,25}$ for single-wall CNTs (SWCNTs) based on the exact Flugge equations will be employed which is more realistic than previous three dimensional shell model $^{24,25}$ and will greatly improve accuracy and enhance the range of applicability as compared with those of the single Donnell equation ${ }^{9}$ and the beam model. ${ }^{16-18}$

\section{THE ANALYSIS METHOD}

In view of the multiple-shell model, ${ }^{10-12}$ each of the nested SWCNT inside a TWCNT is an individual elastic shell, and the adjacent tubes are coupled with each other through the normal vdW interaction without the interlayer friction. It is known that free vibration of a thin cylindrical shell is governed by the exact Flugge dynamic equations, which enjoy improved accuracy and enhanced the range of applicability as compared with the single Donnell equation ${ }^{19}$ and beam model. ${ }^{16-18}$

Now consider a TWCNT with three nested tubes $(k=1,2,3)$ subjected to an initial axial strain $\varepsilon_{x}$. Applying the Flugge equations to the three tubes yields the following nine coupled equations governing the vibration of the axially strained TWCNT: ${ }^{10,11}$

$$
\begin{aligned}
& r_{k}^{2} \frac{\partial^{2} u_{k}}{\partial x^{2}}+\frac{1}{2}(1-\nu) \frac{\partial^{2} u_{k}}{\partial \theta^{2}}+\frac{r_{k}}{2}(1+\nu) \frac{\partial^{2} v_{k}}{\partial x \partial \theta}-\nu r_{k} \frac{\partial w_{k}}{\partial x} \\
& +A_{k} \cdot\left[\frac{1}{2}(1-\nu) \frac{\partial^{2} u_{k}}{\partial \theta^{2}}+r_{k}^{3} \frac{\partial^{3} w_{k}}{\partial x^{3}}-\frac{r_{k}}{2}(1-\nu) \frac{\partial^{3} w_{k}}{\partial x \partial \theta^{2}}\right] \\
& +\varepsilon_{x} \cdot\left(1-\nu^{2}\right) \cdot r_{k}^{2} \cdot \frac{\partial^{2} u_{x}}{\partial x^{2}}=\frac{\rho}{K} \cdot\left(1-\nu^{2}\right) \cdot r_{k}^{2} \frac{\partial^{2} u_{k}}{\partial t^{2}} \\
& \frac{r_{k}}{2}(1+\nu) \frac{\partial^{2} u_{k}}{\partial x \partial \theta}+\frac{r_{k}^{2}}{2}(1-\nu) \frac{\partial^{2} v_{k}}{\partial x^{2}}+\frac{\partial^{2} v_{k}}{\partial \theta^{2}}-\frac{\partial w_{k}}{\partial \theta} \\
& +A_{k} \cdot\left[\frac{3 r_{k}^{2}}{2}(1-\nu) \frac{\partial^{2} v_{k}}{\partial x^{2}}+\frac{r_{k}^{2}}{2}(3-\nu) \frac{\partial^{3} w_{k}}{\partial x^{2} \partial \theta}\right] \\
& +\varepsilon_{x} \cdot\left(1-\nu^{2}\right) \cdot r_{k}^{2} \cdot \frac{\partial^{2} v_{x}}{\partial x^{2}}=\frac{\rho}{K}\left(1-\nu^{2}\right) r_{k}^{2} \frac{\partial^{2} v_{k}}{\partial t^{2}} \\
& \nu r_{k} \frac{\partial u_{k}}{\partial x}+\frac{\partial v_{k}}{\partial \theta}-w_{k}+A_{k} \cdot\left[-r_{k}^{3} \frac{\partial^{3} u_{k}}{\partial x^{3}}+\frac{r_{k}}{2}(1-\nu) \frac{\partial^{3} u_{k}}{\partial x \partial \theta^{2}}\right. \\
& \quad-\frac{R_{k}^{2}}{2}(3-\nu) \frac{\partial^{3} v_{k}}{\partial x^{2} \partial \theta} \\
& +\varepsilon_{x} \cdot\left(1-\nu^{2}\right) \cdot r_{k}^{2} \cdot \frac{\partial^{2} w}{\partial x^{2}}=\frac{1}{K}\left(1-\nu^{2}\right) \cdot r_{k}^{2} \cdot\left[\rho \frac{\partial^{2} w_{k}}{\partial t^{2}}-p_{k}\right] \\
& \left.\frac{\partial^{2} w_{k}}{\partial \theta^{2}}\right]
\end{aligned}
$$

where $u_{k}, v_{k}$ and $w_{k}(k=1,2,3)$ are respectively the longitudinal, circumferential and (inward positive) radial vibration displacements of tube $k ; r_{k}$ is the radius of tube $k$; the subscript $k(=1,2$ and 3$)$ denotes respectively the quantities of the innermost, the middle and the outermost tube; For each tube/layer of the SWCNT, $D$ is the effective bending stiffness, $K$ is the in-plane stiffness, $\rho$ denotes the mass density per unit area on the lateral surface and $A_{k}=D / K \cdot r_{k} \cdot\left(1-\nu^{2}\right)$. We assume that $D, K$ and $\rho$ are the same for the three tubes. It is highlighted that the main difference from most previous studies is that we model each tube in a TWCNT as a two dimensional cylindrical shell. ${ }^{21,22}$ Here the bending and in-plane stiffnesses are the two material constants, which can be measured in experiments or obtained using atomistic simulations. However, they are not necessarily related to the effective wall thickness $h$ and equivalent Young's modules of the one atomlayer SWCNTs. The (inward positive) net radial pressure $p_{k}$ on the $k$-th layer is defined as the sum of the inner pressure due to the $(k-1)$ th layer and outer pressure due to the $(k+1)$ th layer. These quantities can be calculated by: ${ }^{10-12}$

$$
\begin{gathered}
p_{1}=c \cdot\left(w_{2}-w_{1}\right), p_{2}=c \cdot\left(w_{3}-w_{2}\right)-c \cdot \frac{r_{1}}{r_{2}}\left(w_{2}-w_{1}\right) \\
\text { and } p_{3}=-c \frac{r_{2}}{r_{3}}\left(w_{3}-w_{2}\right)
\end{gathered}
$$

where $c$ is the vdW interaction coefficient between adjacent tubes and defined by the second-order derivative of the interlayer potential with respect to the interlayer spacing.

For simply-supported MWCNTs the solution to Eq. (1) can be obtained as

$$
\begin{gathered}
u_{k}(x, \theta, t)=U_{k} \cos \frac{m \pi}{L} x \cdot \cos n \theta \cdot e^{i \omega t} \\
v_{k}(x, \theta, t)=V_{k} \sin \frac{m \pi}{L} x \cdot \sin n \theta \cdot e^{i \omega t} \\
w_{k}(x, \theta, t)=W_{k} \sin \frac{m \pi}{L} x \cdot \cos n \theta \cdot e^{i \omega t}
\end{gathered}
$$

where real numbers $U_{k}, V_{k}$ and $W_{k}$ denote the longitudinal, circumferential and radial displacement amplitudes of tube $k$; $L$ is the length of a TWCNT; $\omega$ and $f=\omega / 2 \pi$ represent respectively the circular and angular frequency; $m$ is the axial half wave number; and $n$ is the circumferential wave number. Substituting Eqs. (3) and (2) into Eq. (1) leads to the following nine homogeneous algebraic equations

$$
\left[M\{(n, \lambda)\}-\Omega^{2} I\right]_{9 \times 9}\left[\begin{array}{c}
U_{1} \\
V_{1} \\
W_{1} \\
\cdots \\
U_{3} \\
V_{3} \\
W_{3}
\end{array}\right]_{9 \times 1}=0
$$


In which $M_{9 \times 9}$ is a $9 \times 9$ matrix whose elements are functions of $n, \lambda$, where $\lambda=L / r_{3} m$ is the normalized axial wavelength (normalized by the outermost diameter of a TWCNT $\left.2 r_{3}\right) ; I$ is a $9 \times 9$ identity matrix and $\Omega^{2}=\omega^{2}-$ $\varepsilon_{x}(K / \rho) \cdot\left(r_{3} \pi / \lambda\right)^{2}$. The condition for the existence of non-zero solutions for $U_{i}, V_{i}$ and $W_{i}(i=1,2, \ldots, N)$ is

$$
\operatorname{det}\left(M-\Omega^{2} I\right)=0
$$

With a given combination of $(\lambda, n)$, Eq. (5) gives 9 eigenvalues $\Omega^{2}$. Here we have three typical cases:

(i) $\varepsilon_{x} \neq 0$, in which the 9 eignevalues $\left(\Omega^{2}\right)$ lead to 9 frequencies $f$ given by $f=\omega / 2 \pi=1 / 2 \pi$. $\sqrt{\Omega^{2}+\varepsilon_{x .}(K / \rho) \cdot\left(r_{3} \pi / \lambda\right)^{2}}$;

(ii) $\varepsilon_{x}=0$, in which the 9 eigenvalues $\left(\Omega^{2}\right)$ give 9 free vibration frequencies $f=\omega / 2 \pi=1 / 2 \pi \cdot \Omega$; and

(iii) $\omega=0$, where the lowest eigenvalues $\left(\Omega^{2}\right)_{\text {min }}$ yields the critical axial buckling strain of a TWCNT as:

$$
\varepsilon_{\mathrm{cr}}=-\frac{\left(\Omega^{2}\right)_{\min }}{\left(\frac{K}{\rho}\right) \cdot\left(\frac{r_{3} \pi}{\lambda}\right)^{2}}
$$

The substitution of an eigenvalue $\Omega^{2}$ into (4) yields the amplitude ratios $U_{k} / W_{3}, V_{k} / W_{3}$ and $W_{k} / W_{3}(k=1,2,3)$, defining the vibration or initial axial buckling mode of a TWCNT associated with $f$ or $\varepsilon_{\mathrm{cr}}$. In particular, it is noted that the lowest fundamental frequency also corresponds to $\left(\Omega^{2}\right)_{\min }$. Thus, the vibration mode associated with the lowest fundamental frequency should be identical to the initial axial buckling mode of the TWCNT. Here it should be pointed out that continuum mechanics models, e.g., elastic shell models, can only capture the low frequency vibrations of CNTs with relatively long wavelength (or small wave numbers). These vibrations can be found at the bottom of the phone-dispersion diagram of SWCNTs predicted by atomistic simulations. ${ }^{27-28}$ The most recent study ${ }^{29}$ further indicates that, even in the range of low frequency, while the continuum models math the atomistic simulations for extensional vibration modes they break down for inextensional modes (i.e., axial half wave number is equal to zero) with sufficiently large circumferential wave number. Thus, caution must be taken when using the continuum models for the vibration of CNTs. Specifically, it is essential to justify the validity of the continuum models by comparing them with more realistic atomistic models ${ }^{27-30}$ or experiments. In previous studies, the present multiple-shell model has been employed for a series of buckling ${ }^{9,23-26}$ and vibration ${ }^{9-12,22}$ analyses of CNTs with a reasonably good or excellent agreement with the available experiments, ${ }^{23,25}$ molecular dynamics simulations,,${ }^{9,10,25}$ lattice dynamics models ${ }^{12,22}$ and molecular mechanics models. ${ }^{25}$ In particular, the present work is focused on the low frequency extensional vibration of TWCNTs with a nonzero axial half wave number, where, as shown in Ref. [29], the continuum models serve as a good approximation for CNTs. Therefore, it is anticipated that the present multiple-shell model can be efficiently used to give a reliable description to the low frequency extensional vibration of strained TWCNTs.

\section{RESULTS AND DISCUSSION}

In what follows, we will apply the method presented in Section 2 to examine the vibration of axially strained triple-wall CNTs. The vibration behavior of multi-wall CNTs changes significantly with the variation of their radii. ${ }^{10,11}$ For this reason, two types of TWCNTs will be considered in the present study, i.e., large radius TWCNTs of the innermost radius $r_{1}=5 \mathrm{~nm}$ and small radius TWCNTs of $r_{1}=1 \mathrm{~nm}$. For each case, to further investigate the influence of their geometric size in the axial direction we will consider the TWCNTs with the normalized axial wavelength $\left(\lambda=L / r_{3} m\right)$ increasing from $5,10,20$ to 30 . The values of the material constants used in the present study for each layer of TWCNTs are $D=2 \mathrm{eV},{ }^{12} K=360 \mathrm{~J} / \mathrm{m}^{2}$ and $\rho=\left(2.27 \mathrm{~g} / \mathrm{cm}^{3}\right) \times$ $0.34 \mathrm{~nm}^{9-12}$ The value of the interlayer vdW interaction coefficient is $c=101 \mathrm{GPa} / \mathrm{nm} \cdot{ }^{10,25}$

\subsection{Case 1: TWCNTs with a Large Innermost Radius (5 nm)}

The vibration spectra for this case have been plotted in Figure 1 for the free TWCNT (i.e., $\varepsilon_{x}=0$ ) with the normalized axial wavelength $\lambda$ fixed at 5 and the circumferential wave number $n$ rising from 0 to 10 . Nine frequency curves are shown in Figure 1, and, from the lowest frequency to the highest one, are referred to as $f_{1}, f_{2}, \ldots, f_{9}$, respectively. These frequency curves are associated with

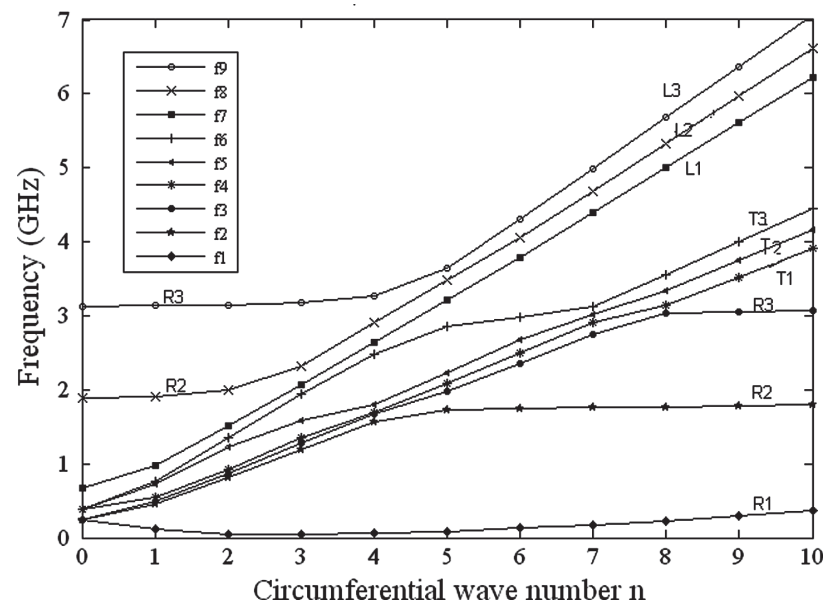

Fig. 1. The nine free vibration frequencies of Case $1\left(\varepsilon_{x}=0\right)$ with the normalized axial wavelength $\lambda=5$. Here $f_{1}<f_{2}<\cdots<f_{9}$. $\left(L_{1}, L_{2}, L_{3}\right)$, $\left(T_{1}, T_{2}, T_{3}\right)$ and $\left(R_{1}, R_{2}, R_{3}\right)$ denote various torsional, longitudinal and radial vibration modes of Case 1 associated with corresponding frequency at a specific range of $n$. 
different vibration modes, e.g., three torsional modes $T_{1}$, $T_{2}$ and $T_{3}$, three longitudinal modes $L_{1}, L_{2}$ and $L_{3}$, and three radial modes $R_{1}, R_{2}$ and $R_{3}$, as shown in Figure 1 for a specific range of $n$.

It is noted in Figure 1 that with the variation of $n$, the vibration mode associated with the same frequency curve could transform from one mode to another. For example, the highest frequency $f_{9}$ is associated with $R_{3}$ mode for $n \leq 3$; it corresponds to $T_{3}$ mode for $n \geq 6$; while for $4 \leq$ $n<6$, it is associated with a combined mode with comparable radial and torsional displacements. On the other hand, one radial mode of the TWCNT could correspond to different frequency curves when the value of $n$ falls in different areas. For instance, $R_{3}$ mode corresponds to the highest frequency $f_{9}$ for $n \leq 3$, but to frequency $f_{3}$ when $n \geq 8$. However, for $5 \leq n \leq 7$, this intertube radial mode $R_{3}$ with predominant radial displacements disappears. Only the torsional or combined modes with coupling between radial and longitudinal/torsional vibrations can be found for $f_{3}$ to $f_{9}$. Similar phenomenon is also observed in Figure 1 for $R_{2}$ mode of the TWCNT. It should be pointed out that the radial modes of MWCNTs predicted in Ref. [19] cover the whole range of $n$ considered in the work. This is in general not true because torsional and longitudinal displacements are completely ignored in the single Donnell equation used. Thus, in the range of $n$ where the radial mode of CNTs does not exist, the frequency given by the single Donnell equation with only a radial displacement becomes physically less meaningful.

Next, an axial strain $\varepsilon_{x}$ is applied to the current case with the ratio $q=-\varepsilon_{x} / \varepsilon_{\text {cr }}$ increasing from -0.8 (compressive strain) to 0.8 (tensile strain). For each given value of $\varepsilon_{x}\left(=q \cdot \varepsilon_{\mathrm{cr}}\right)$ nine frequency curves generally similar to those in Figure 1 can be obtained for the strained TWCNT, except that some of the frequency curves deviate from their original positions in Figure 1. This shows that the frequency shift is due to the applied axial strain. In spite of this, our analysis of amplitude ratios indicates that the vibration modes of the current strained case associated with all the nine frequency curves are basically the same as those shown in Figure 1. This finding of the strain effect on vibration modes is consistent with the previous results obtained for the two special cases, i.e., the transverse bending ${ }^{16}$ and radial vibrations, ${ }^{19}$ of multiwall CNTs. It should be kept in mind that the initial axial strain has no significant influence on the vibration deformation patterns. Then we focus our attention on the axial strain-induced frequency changes of these vibration modes.

The frequency shift $\Delta f_{k}=f_{k}-\bar{f}_{k}(k=1,2, \ldots, 9)$ is calculated, where $f_{k}$ is the frequency of free vibration in Figure 1 and $\bar{f}_{k}$ is the corresponding frequency under an initial axial strain $\varepsilon_{x}$. The results show that the axial straininduced changes can almost be neglected for the highest three frequencies $\left(f_{7}, f_{8}\right.$ and $\left.f_{9}\right)$. These mainly correspond to $T_{1}, T_{2}$ and $T_{3}$ modes of Case 1 (Fig. 1), i.e., the torsional

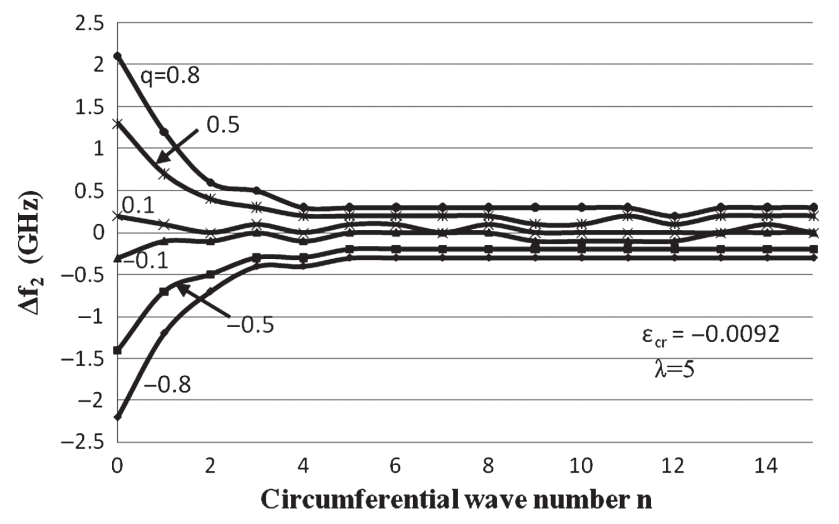

Fig. 2. The axial strain induced frequency shift $\Delta f_{2}$ obtained for Case 1 with the normalized axial wavelength $\lambda=5$ and $n=0$ to 15 . Here coefficient $q=-\varepsilon_{x} / \varepsilon_{\mathrm{cr}}$. The positive $q$ gives the tensile axial strain $\varepsilon_{x}=q \varepsilon_{\mathrm{cr}}$ and the negative one leads to the compressive axial strain.

vibration of the individual layers, and $R_{2}$ and $R_{3}$ (Fig. 1), i.e., the two intertube radial vibrations, where some of adjacent tubes are moving in the opposite directions. The detectable changes are observed for the five lower frequencies $\left(f_{2}, f_{3}, f_{4}, f_{5}\right.$ and $\left.f_{6}\right)$. To shed some lights on this, the frequency shift of $f_{2}$ is calculated in Figure 2 as an example where the normalized axial wavelength $\lambda$ is fixed at 5 . As expected, Figure 2 shows that $f_{2}$ upshifts under the tensile stain $(q>0)$ but downshifts when the compressive strain $(q<0)$ is applied. In particular, the magnitude of the frequency shift $\left|\Delta f_{2}\right|$ grows significantly with the increasing initial axial strain.

However, for a given $\varepsilon_{x},\left|\Delta f_{2}\right|$ decreases rapidly when $n$ rises from 0 to 4 , and remains almost constant when $n \geq 5$. For the axisymmetric case with $n=0$, the maximum $\left|\Delta f_{2}\right| \approx 2 \mathrm{GHz}$ is found at $\varepsilon_{x}= \pm 0.8 \varepsilon_{\mathrm{cr}}= \pm 0.72 \%$. Since $f_{2} \approx 250 \mathrm{GHz}$ for $n=0$ (see Fig. 1) the corresponding relative change $\left|\Delta f_{2}\right| / f_{2}$ is only $0.9 \%$ with $\lambda=5$, which, based on our results, will further reduce when the normalized wavelength $\lambda$ is raised from 5 to 10,20 and 30 . A similar but less pronounced effect of the initial axial strain is obtained for higher frequencies from $f_{3}$ to $f_{6}$. It is noted that the five frequency curves $\left(f_{2}\right.$ to $\left.f_{6}\right)$ in Figure 1 are primarily associated with the combined vibration modes for $n<4$ or 6 , the longitudinal modes $L_{1}, L_{2}$ and $L_{3}$ of the three individual tubes for $n \geq 7$ and the two interlayer radial modes $R_{2}$ and $R_{3}$ for $n \geq 5$ and $n \geq 8$, respectively. Thus, the above results for $f_{2}$ to $f_{6}$ indicate that the frequencies of the longitudinal modes and intertube radial modes obtained for large $n$ in Figure 1 are generally insensitive to the initial axial strain. The strong effect of the initial axial strain occurs for the fundamental frequency $f_{1}$, which usually corresponds to a radial mode $R_{1}$ for $n \geq 2$. In this radial mode $R_{1}$, the three constituent layers are vibrating collectively in the same direction and with almost the same radial displacement.

For a detailed study of $f_{1}, \Delta f_{1}$ is calculated in Figure 3 as a function of the initial axial strain $\varepsilon_{x}$ and 


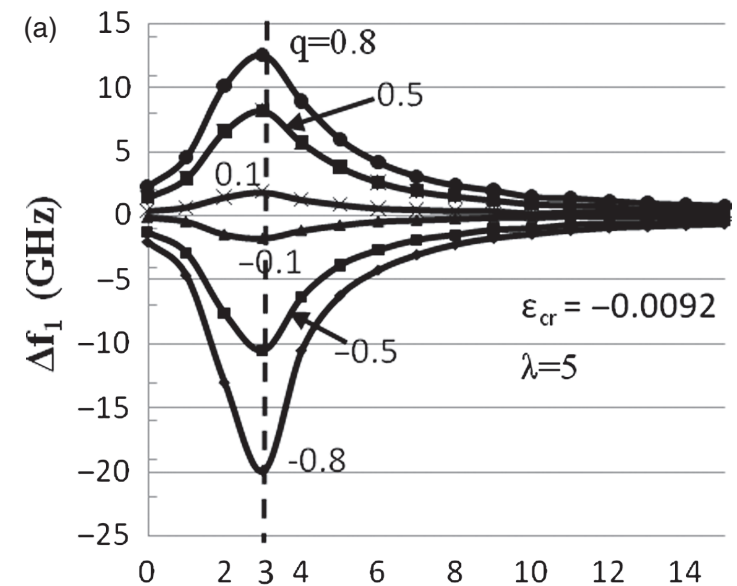

Circumferential wave number $n$

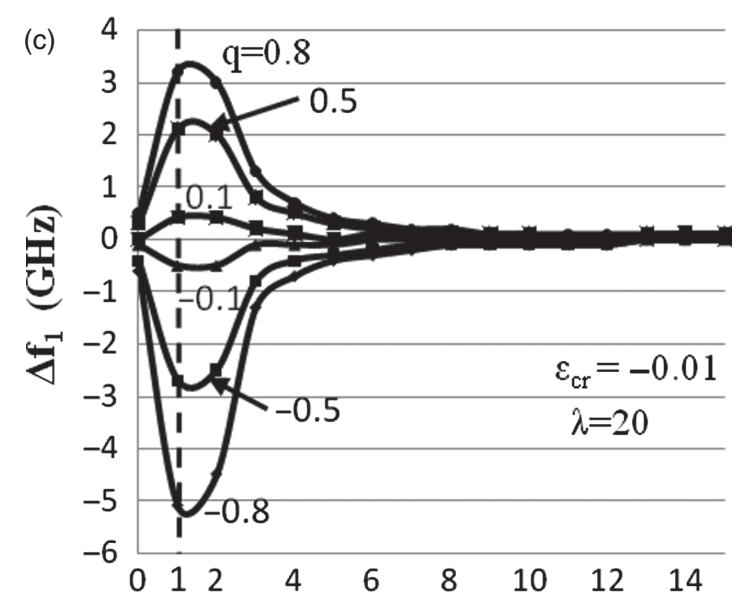

Circumferential wave number $n$
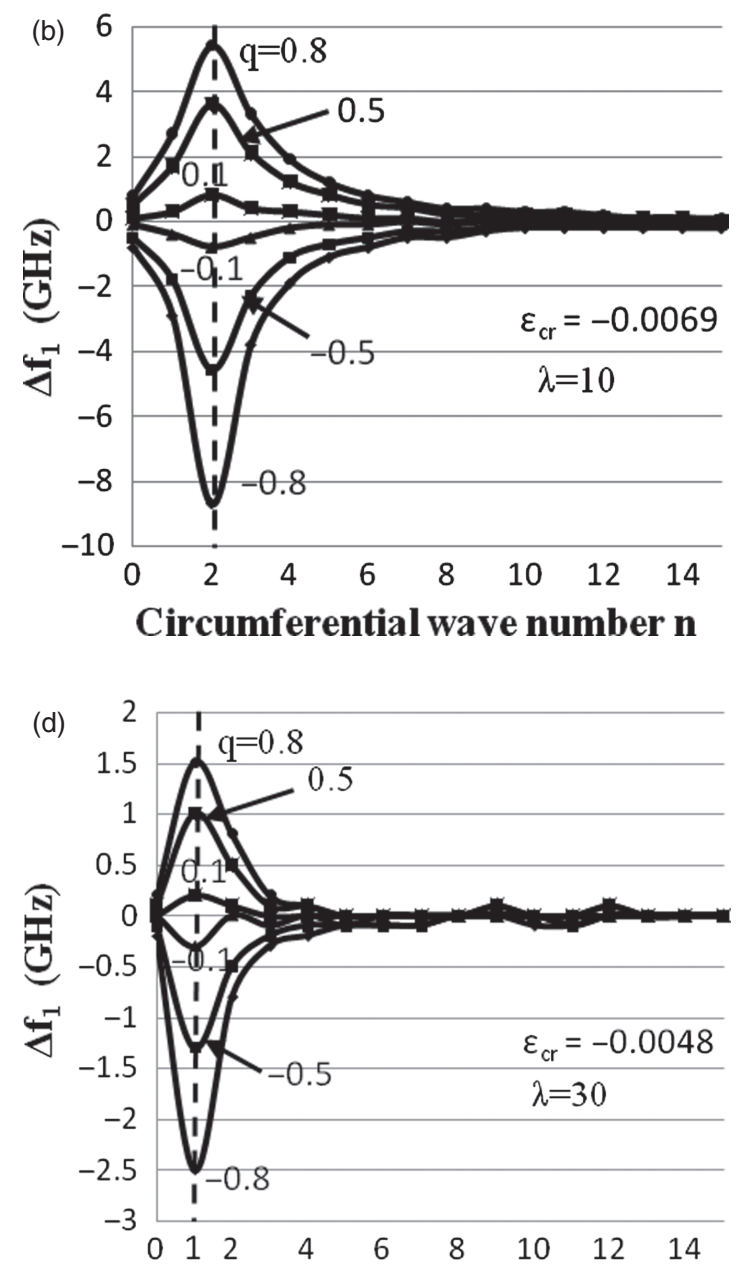

Circumferential wave number $n$

Fig. 3. The axial strain induced frequency shift $\Delta f_{1}$ obtained for Case 1 with $n=0$ to 15 and the normalized axial wavelength $\lambda$ equal to (a) 5 , (b) 10 , (c) 20 and (d) 30 , respectively. Here coefficient $q=-\varepsilon_{x} / \varepsilon_{\mathrm{cr}}$. The positive $q$ gives the tensile axial strain $\varepsilon_{x}=q \varepsilon_{\mathrm{cr}}$ and the negative one leads to the compressive axial strain.

the circumferential wave number $n$. As can be seen in Figure 3(a) with $\lambda=5, f_{1}$ increases (i.e., $\Delta f_{1}>0$ ) with enhanced tensile strain but decreases (i.e., $\Delta f_{1}<0$ ) with increasing compressive strain, which is analogous to those shown in Figure 2 for $f_{2}$. On the other hand, while $\left|\Delta f_{2}\right|$ decreases monotonically with $n$ (see Fig. 2), $\left|\Delta f_{1}\right|$ increases first with the growing $n$ and reaches its maximum value at $n=3$ for all the axial strains considered. It then decreases with $n$ and approaches to zero when $n$ is sufficiently large. In Figure 3(a), the maximum value obtained for $\left|\Delta f_{1}\right|$ is $13 \mathrm{GHz}$ under a tensile strain $\varepsilon_{x}=0.8 \varepsilon_{\mathrm{cr}}=0.73 \%$ and $20 \mathrm{GHz}$ under a compressive strain $\varepsilon_{x}=-0.8 \varepsilon_{\mathrm{cr}}=-0.73 \%$. The corresponding relative changes $\left|\Delta f_{1}\right| / f_{1}$ are calculated as $34 \%$ and $53 \%$, respectively. Relative changes above $25 \%$ can be obtained in the neighborhood of $n=3$, i.e., $2 \leq n \leq 4$. Beyond this small range of $n,\left|\Delta f_{1}\right|$ becomes small or negligible. In this case, the maximum frequency shift and relative change are much greater than $2 \mathrm{GHz}$ and $0.9 \%$ obtained for $f_{2}$ under the same strain (Fig. 2), showing that the fundamental frequency $f_{1}$ mainly associated with $R_{1}$ mode is much more sensitive to the initial axial strain.

In addition, it is noted that a compressive axial strain alters frequency $f_{1}$ more efficiently than a tensile strain does. Analogous behavior is observed in Figure 3 (b), (c) and $d$ with $\lambda=10,20$ and 30, respectively. More specifically, when $\lambda$ increases from 5, to 10, 20 or even larger, the value of $n$ corresponding to the maximum $\left|\Delta f_{1}\right|$ decreases from $n=3$ to 2 and to 1 , and the range of $n$ associated with a large relative change, say above $20 \%$, narrows down from $2 \leq n \leq 4$ to $n=1$. In this process, the vibration mode associated with the maximum $\left|\Delta f_{1}\right|$ transforms from the radial mode $R_{1}$ with 3 or 2 circumferential waves to the transverse bending modes with a circular cross-section.

It is worth mentioning that, for any given $\varepsilon_{x}$, the maximum $\left|\Delta f_{1}\right|$ is always achieved for the minimum value of the fundamental frequency $f_{1}$, i.e., corresponding to the lowest eigenvalue of Eq. (5) (see Fig. 1 for example). As stated in Section 2, such a vibration mode is identical to the initial axial buckling mode of the TWCNT, where 


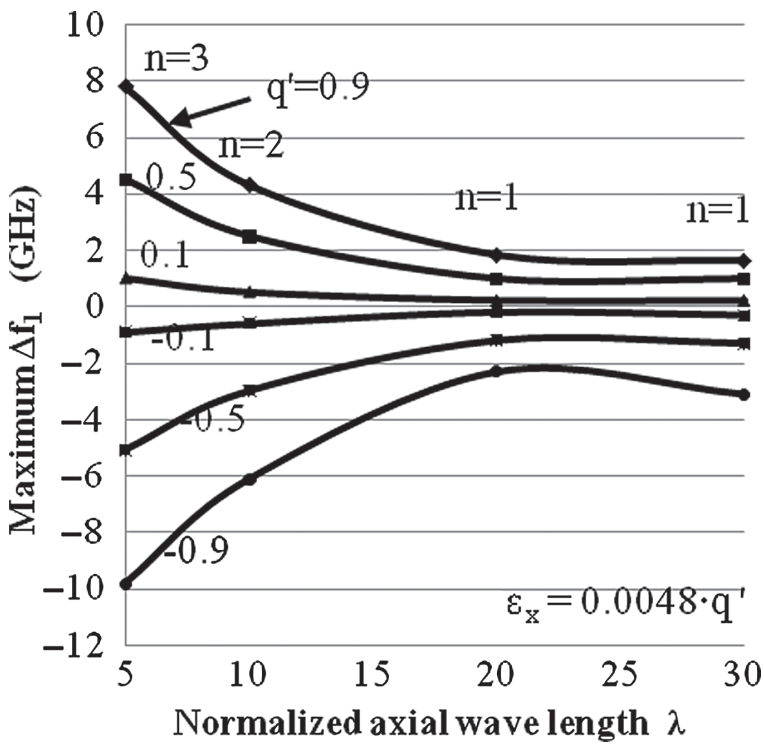

Fig. 4. The maximum $\Delta f_{1}$ obtained for example 1 (associated with the lowest value of $f_{1}$ in Fig. 1) with the normalized axial wavelength $\lambda$ varying from 5 to 30 . Here $\varepsilon_{x}=0.0048 q^{\prime}$. The positive coefficient $q^{\prime}$ gives the tensile axial strain and the negative one leads to the compressive axial strain.

the axial loadings can most efficiently affect the structural rigidity of the TWCNT.

To further study the $\lambda$-dependence of the strain effect, the maximum value of $\left|\Delta f_{1}\right|$ at $\varepsilon_{x}=0.0048 q^{\prime}\left(q^{\prime}= \pm 0.1\right.$, \pm 0.5 and \pm 0.9 ), is shown in Figure 4 with $\lambda$ rising from 5 to 30 . The value of $n$ associated with the maximum $\left|\Delta f_{1}\right|$ is also presented in the figure. It is found that in addition to the transformation of the vibration modes due to the variation of $\lambda$, the value of maximum $\left|\Delta f_{1}\right|$ at the same $\varepsilon_{x}$ decreases significantly with increasing $\lambda$ when $\lambda \leq 20$. For example, with the axial strain $\pm 0.43 \%$, i.e., $q= \pm 0.9$, the maximum $\left|\Delta f_{1}\right|$ is $8 \mathrm{GHz}$ at $\lambda=5$ but reduces four times to less than $2 \mathrm{GHz}$ at $\lambda=20$. After that the strain-induced frequency shift becomes less sensitive to the normalized axial wavelength.

\subsection{Case 2: TWCNTs with a Small Innermost Radius (1 nm)}

The free vibration frequencies of this case are plotted in Figure 5 with the normalized axial wavelength $\lambda=5$. Again nine frequency curves are obtained in Figure 5 for this TWCNT, which are denoted by $f_{1}, f_{2}, \ldots, f_{9}$, respectively. Here, different from the large radius case discussed before, the coupling between the vibration of the adjacent layers are less pronounced, especially for large $n$, say $n>5$. This can be explained by the fact that the effect of the interlayer vdW force is weak as compared with the high structural rigidity due to the small radius. Accordingly, in Figure 5, the eight frequency curves $\left(f_{2}, f_{3}, \ldots, f_{9}\right)$ primarily represent the frequencies of two radial $\left(R_{2}, R_{3}\right)$ modes, three longitudinal $\left(L_{1}, L_{2}, L_{3}\right)$ modes and three torsional $\left(T_{1}, T_{2}\right.$, $T_{3}$ ) modes of the individual layers. The $R_{1}$ mode associated with $f_{1}$ usually represents the collective radial

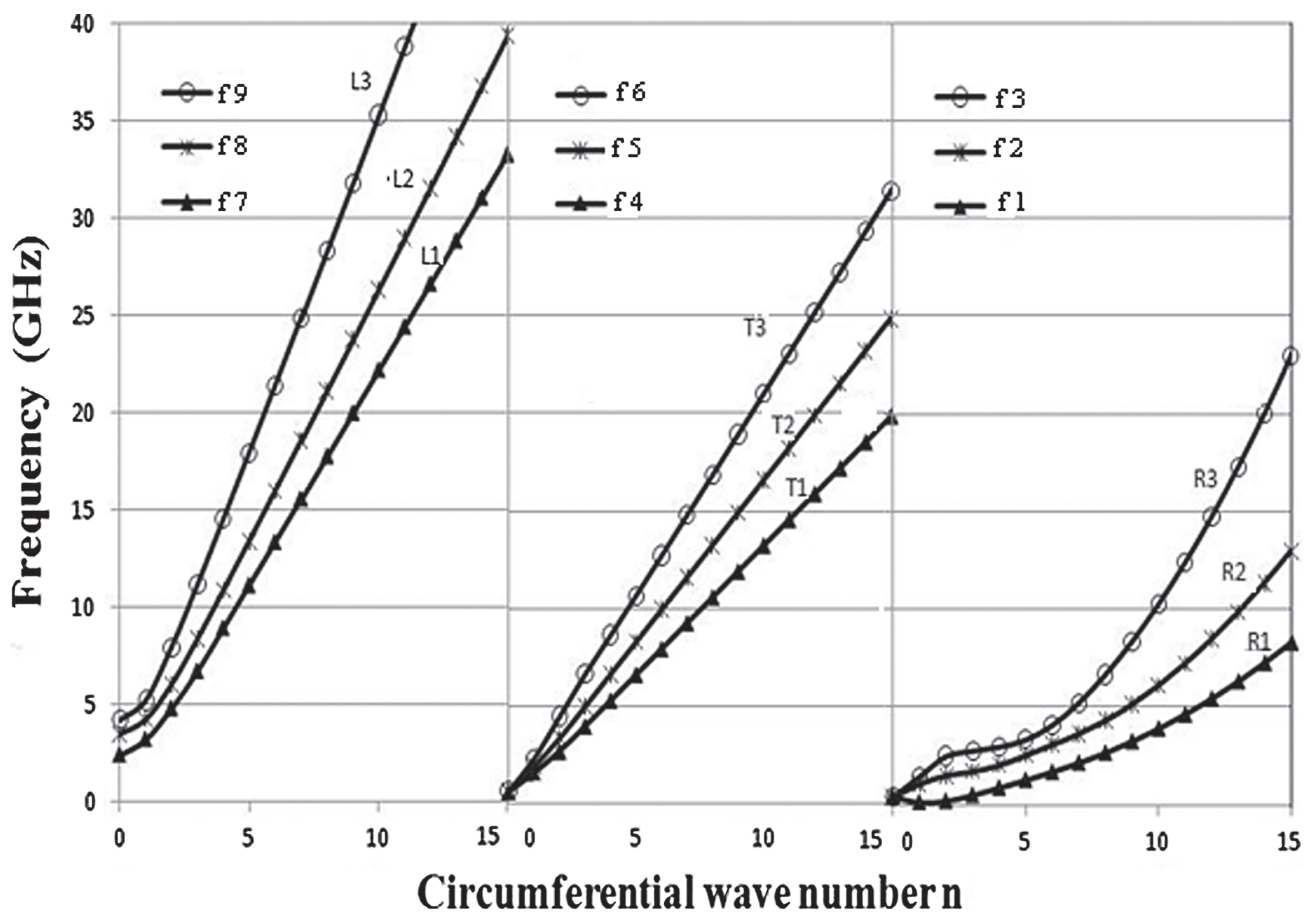

Fig. 5. The nine free vibration frequencies of example $2\left(\varepsilon_{x}=0\right)$ with the normalized axial wavelength $\lambda=5$. Here $f_{1}<f_{2}<\cdots<f_{9}$. $\left(L_{1}, L_{2}, L_{3}\right)$, $\left(T_{1}, T_{2}, T_{3}\right)$ and $\left(R_{1}, R_{2}, R_{3}\right)$ denote various torsional, longitudinal and radial vibration modes of Case 1 associated with corresponding frequency at a specific range of $n$. 
vibration where the three layers are vibrating with almost an identical displacement. The intertube radial modes are only available to frequencies $f_{2}$ and $f_{3}$ for small $n$, say, $n<5$ with weak coupling between adjacent layers.

It is found in our analysis that $f_{7}, f_{8}, f_{9}$ and $f_{4}$, $f_{5}, f_{6}$, associated mainly with torsional and longitudinal vibrations, are insensitive to the initial axial strain, whereas $f_{1}, f_{2}, f_{3}$, primarily corresponding to radial or transverse bending ( $f_{1}$ with $n=1$ and $\lambda \geq 10$ ) vibrations, can be significantly altered by applying an initial axial strain. The tendency of frequency shift with respect to the changes in $\varepsilon_{x}$ and $n$ is shown in Figures 6(a) and (b) $(\lambda=5)$ for $f_{2}$ and $f_{3}$, respectively. For the largest strain $\varepsilon_{x}= \pm 2.6 \% \quad(q= \pm 0.8)$ in Figure 6, the maximum change around $25 \mathrm{GHz}$ is obtained for both $f_{2}$ and $f_{3}$ at $n=0$, corresponding to the relative changes $\left(\left|\Delta f_{2}\right| / f_{2},\left|\Delta f_{3}\right| / f_{3}\right)$ measuring to $3.6 \%$.

It should be noted that the maximum stain $\varepsilon_{x}= \pm 2.6 \%$ considered in Figure 6 is 3.6 times the maximum strain $\pm 0.73 \%$ acting on Case 1 in Figure 2. Taking this into consideration we see that the maximum relative change of $3.6 \%$ is comparable with $0.9 \%$ obtained for $f_{2}$ of Case 1 but the maximum frequency shift $25 \mathrm{GHz}$ is much greater than $2 \mathrm{GHz}$ shown in Figure 2. In Figure 6, although the frequency shift tends to decrease with increasing $n$, the
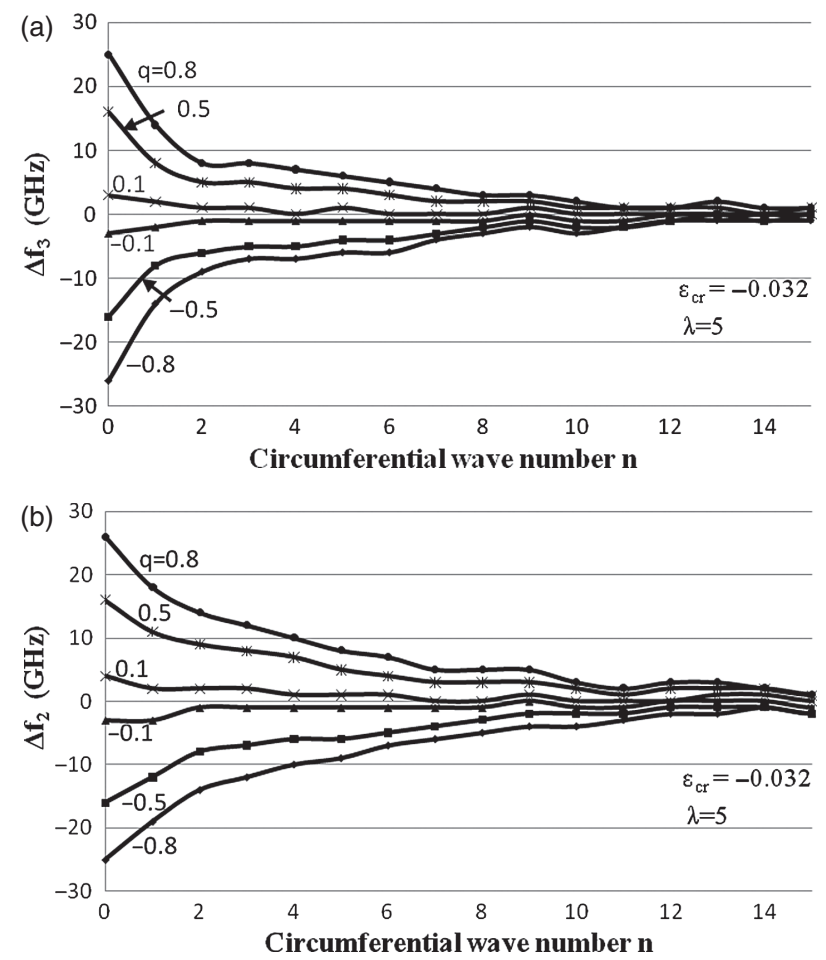

Fig. 6. The axial strain induced frequency shift (a) $\Delta f_{3}$ and (b) $\Delta f_{2}$ obtained for Case 2 with the normalized axial wavelength $\lambda=5$ and $n=0$ to 15 . Here coefficient $q=-\varepsilon_{x} / \varepsilon_{\mathrm{cr}}$. The positive $q$ gives the tensile axial strain $\varepsilon_{x}=q \varepsilon_{\mathrm{cr}}$ and the negative one leads to the compressive axial strain. significant change of $f_{2}$ and $f_{3}$ can be observed for $n \leq$ 6 . However, further raising $n$ leads to a small or negligible frequency shift. Similar to Case 1, the most significant effect of initial axial strain again occurs for $f_{1}$. The axial strain-induced shift of $f_{1}$ is thus presented for this TWCNT in Figure 7 (a), (b), (c) and (d) with $\lambda=5,10$, 20 and 30, respectively. The tendency of frequency to shift with $\varepsilon_{x}$ and $(n, \lambda)$ in Figure 7 is similar to that shown in Figure 3. The maximum values of $\left|\Delta f_{1}\right|$ is achieved at $n=2$ and $\lambda=5$ (Fig. 7(a)), and $n=1$ and $\lambda=10$, 20 and 30 (Figs. 7(b), (c) and (d)), which corresponds to radial vibration $R_{1}$ with $n=2$ and the transverse bending vibration with $n=1$. In particular, these vibration modes correspond to the lowest value of $f_{1}$ associated with the smallest eigenvalue of Eq. (5) (see Fig. 5 for example). In other words, the frequency becomes most sensitive to the initial axial strain when Case 2 is vibrating in the lowest frequency associated with the deformation pattern identical to its axial buckling mode. In Figure 7(a), the maximum frequency shifts of $80 \mathrm{GHz}$ and $130 \mathrm{GHz}$ are obtained for $n=2$ and $\varepsilon_{x}= \pm 2.6 \%$. The relative changes $\left|\Delta f_{1}\right| / f_{1}$ are calculated as $36 \%$ and $55 \%$, respectively. It is seen that the maximum relative changes of $f_{1}$ are almost the same as those obtained for Case 1 in Section 3.1 but the maximum $\Delta f_{1}$ at $\varepsilon_{x}= \pm 2.6 \%$ (Fig. 6) are around seven times those of Case 1 at $\varepsilon_{x}= \pm 0.72 \%$ (Fig. 2). From this point of view, the same axial strain would lead to a greater frequency shift for the small radius case compared with the large radius case studied before.

We further examine the $\lambda$-dependence of axial strain effect. The maximum of $\left|\Delta f_{1}\right|$ is calculated in Figure 8 with $\varepsilon_{x}=0.0036 q^{\prime}\left(q^{\prime}= \pm 0.1, \pm 0.5\right.$ and \pm 0.9$)$ and $\lambda$ increasing from 5 to 30 . Again, the corresponding value of $n$ is also shown in the figure to specify the vibration modes associated with the maximum $\left|\Delta f_{1}\right|$. In Figure 8, the maximum $\left|\Delta f_{1}\right| 12 \mathrm{GHz}$ is achieved at $\lambda=5$. The value then decreases with rising the wavelength and reduces by $50 \%$ to $6 \mathrm{GHz}$ at $\lambda=10$. For $\lambda \geq 10$, the influence of the axial wavelength becomes less pronounced. These results are consistent with those obtained for Case 1 in Figure 4. It then follows that the same initial axial strain will generate a greater change in the fundamental frequency of TWCNTs with a shorter normalized axial wavelength.

It is well known that, in longitudinal and torsional vibrations, the constituent tubes of TWCNTs are vibrating with predominant in-plane displacements. Their frequencies are therefore mainly determined by the in-plane extension stiffness and Poisson ratio, which are material constants (See Section 2) that cannot be significantly changed by external loadings. The dependence of the material constants on the initial axial strain is thus ignored in the present study, which naturally yields negligible effect of the initial axial strain on the longitudinal and torsional vibrations.

In addition, our analysis shows that the frequency of the intertube radial modes, i.e., $R_{2}$ and $R_{3}$ of Case 1 , are 


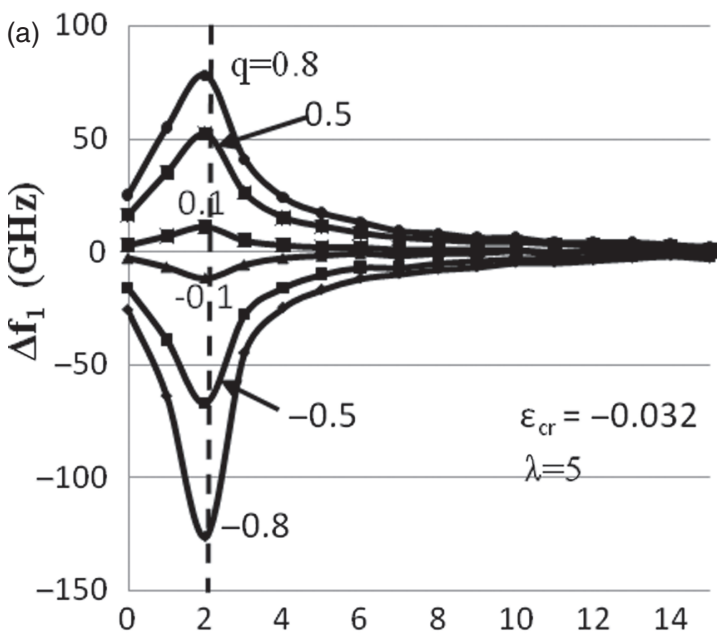

Circumferential wave number $n$

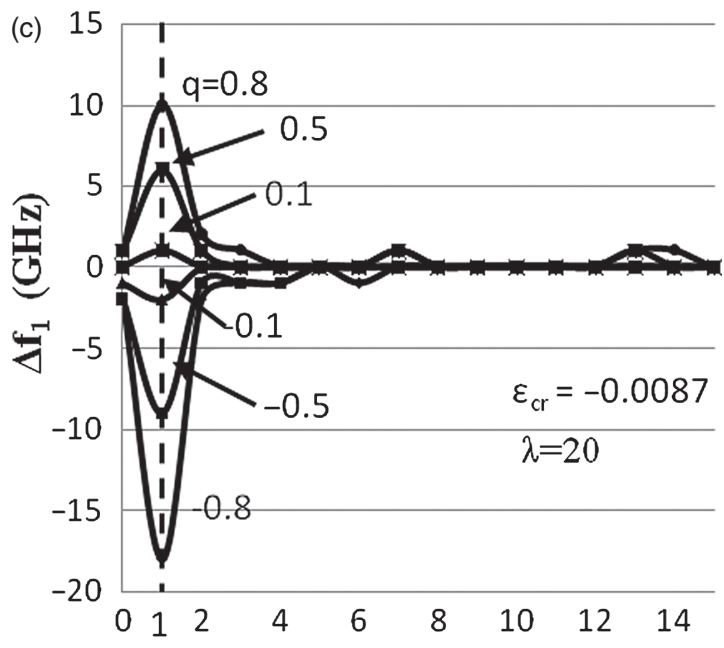

Circumferential wave number $n$

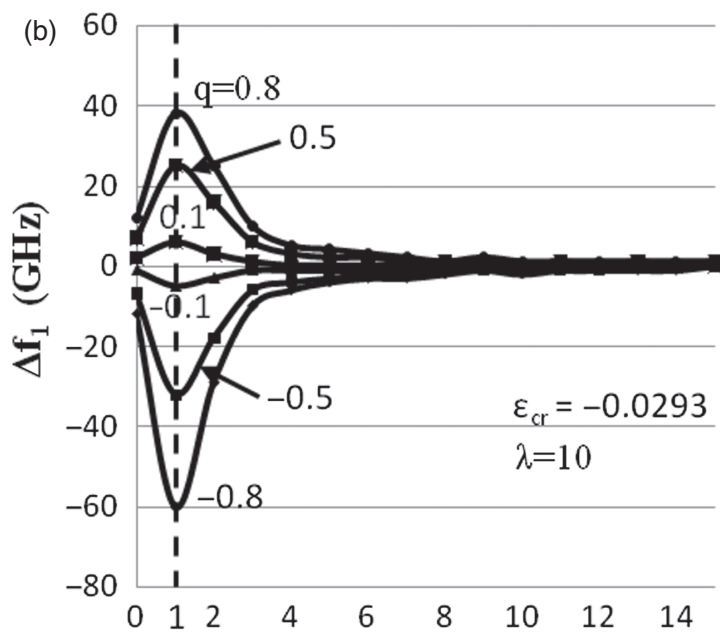

Circumferential wave number $n$

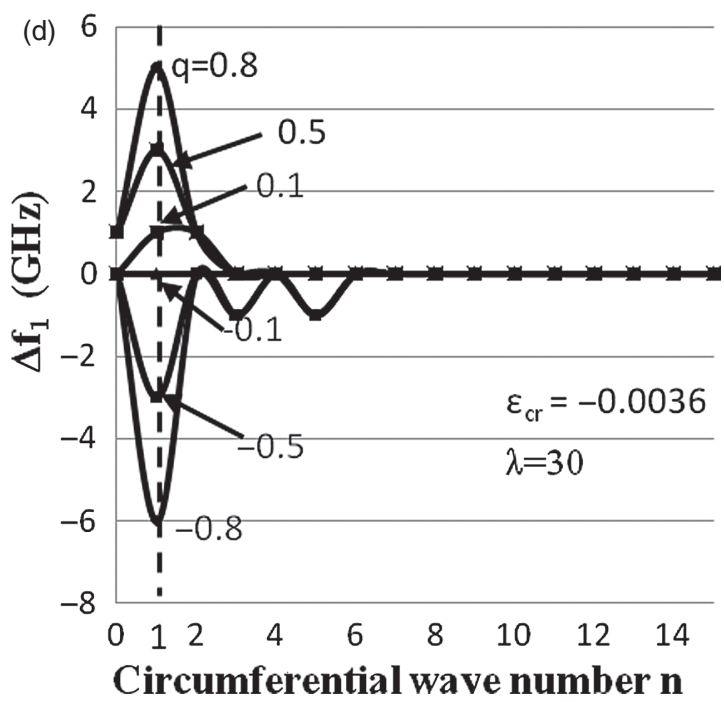

Fig. 7. The axial strain induced frequency shift $\Delta f_{1}$ obtained for Case 2 with $n=0$ to 15 and the normalized axial wavelength $\lambda$ equal to (a) 5 , (b) 10 , (c) 20 and (d) 30 , respectively. Here coefficient $q=-\varepsilon_{x} / \varepsilon_{\mathrm{cr}}$. The positive $q$ gives the tensile axial strain $\varepsilon_{x}=q \varepsilon_{\mathrm{cr}}$ and the negative one leads to the compressive axial strain.

mainly determined by the interlayer vdW interaction characterized by the vdW interaction coefficient $c$, which is a function of the interlayer spacing. ${ }^{10}$ This interaction is equivalent to a linear spring with an elastic coefficient $c$ that primarily determines the vibration frequencies of the system. In the current work, the applied axial strain is not high enough to substantially alter the interlayer spacing and the value of $c$. This leads to the weak effect of the initial axial strain on the frequencies of the intertube radial or transverse modes observed in the present study and some previous studies ${ }^{16,19}$ as well. For small $n$, a stronger axial strain effect on the $R_{2}$ and $R_{3}$ modes of Case 2 (see Fig. 6) is observed because the effect of the vdW interaction in Case 2 is weaker than that in Case 1. The higher frequencies and smaller off-plane displacements of the intertube radial vibrations could also contribute to the small axial strain effect on these modes. On the other hand, in the radial modes or transverse bending mode associated with the lowest frequency $f_{1}$, the off-plane radial or transverse displacement predominates and all the constituent layers have nearly the same displacement, i.e., the interlayer vdW interaction is not significantly involved in these modes.

The structural rigidity of the TWCNTs therefore plays an essential role in determining the frequencies of these modes. The tensile or compressive axial strain is able to enhance or decrease the structural rigidity and thus, results in the large frequency shift of TWCNTs. This accounts for the strong axial strain effect on the $R_{1}$ mode of the TWCNTs. In the extreme case when the compressive strain is equal to the critical axial strain, the buckling of TWCNTs will occur and the structural rigidity as well as the frequency of the TWCNTs will go to zero. Further, it is noted that even for the fundamental frequency, the large frequency shift is only observed for a few lowest 


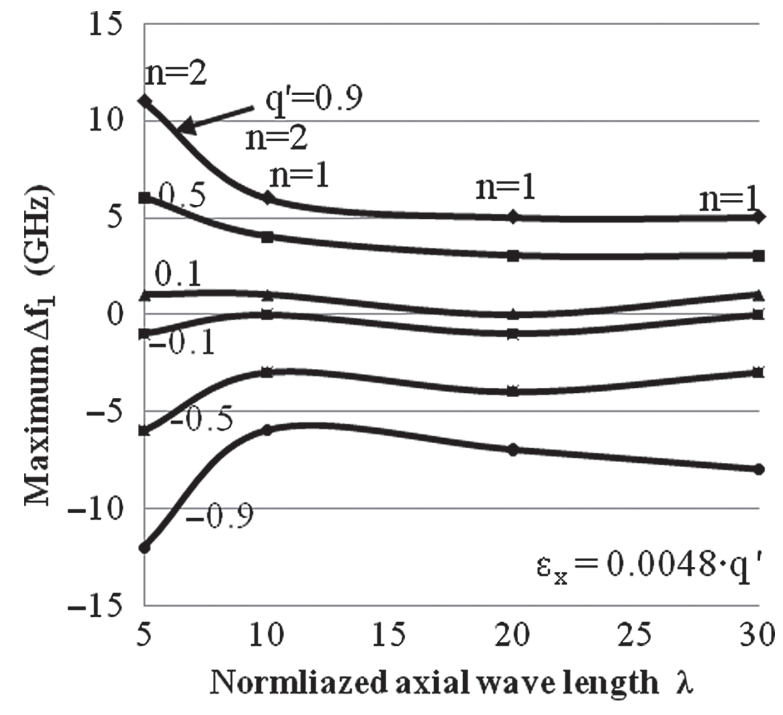

Fig. 8. The maximum $\Delta f_{1}$ obtained for Case 2 (associated with the lowest value of $f_{1}$ in Fig. 5) with the normalized axial wavelength $\lambda$ varying from 5 to 30 . Here $\varepsilon_{x}=0.0036 q^{\prime}$. The positive coefficient $q^{\prime}$ gives the tensile axial strain and the negative one leads to the compressive axial strain.

values with small $n$; whereas the frequency shift becomes negligible for higher frequencies associated with large $n$. This is simply because the large value of $n$ is associated with a higher frequency and naturally, a smaller radial or transverse displacement.

\section{CONCLUSIONS}

The two dimensional shell model for SWCNTs based on the exact Flugee shell theory is employed to conduct a comprehensive study on the vibration properties of TWCNTs subject to an initial axial strain. The emphasis is placed on the axial strain effect on the vibration frequency of TWCNTs. To this end, the dependence of the frequency shift on the axial strain is quantified for TWCNTs with innermost radius of $5 \mathrm{~nm}$ and $1 \mathrm{~nm}$, respectively. The influence of the normalized axial wavelength $\lambda$ on the initial axial strain effect is also examined in detail. In addition, effort has been made to achieve an in-depth physical understanding of the obtained numerical results. The major conclusions of the present study are summarized as follows:

1. The torsionl and longitudinal vibrations or intertube radial vibrations of TWCNTs are largely controlled by in-plane extension stiffness and Poisson ratio, or the intertube vdW interaction coefficient, which cannot be significantly changed by the initial axial strain. The frequencies of these modes are therefore insensitive to an initial axial strain.

2. A tensile or compressive axial strain strengthens or weakens the structural rigidity of TWCNTs and thus, significantly up-shifts or down-shifts the frequencies of the vibration modes with a substantial radial/transverse displacement, such as fundamental radial modes, transverse bending modes and some of the combined modes, in the range of small circumferential wave number, e.g., $n=0$ to 5 .

3. The most significant effect of the axial strain occurs for the lowest fundamental frequency that is associated with the vibration modes identical to the initial axial buckling mode. With the normalized axial wavelength increasing from 5 to 10 and 20 or larger, this vibration mode transforms from the radial modes with $n=3$ to 2 , to the transverse bending mode with $n=1$.

4. The effect of initial axial strain appears to be more pronounced for a small radius TWCNTs (e.g., the innermost radius $1 \mathrm{~nm}$ ) vibrating with a short axial wavelength (e.g., 5 times of the outermost radius) than a large radius ones (e.g., the innermost radius $5 \mathrm{~nm}$ ) oscillating with a long axial wavelength (e.g., more than 10 times of the outermost radius). Hence, small radius TWCNT with short characteristic axial length enjoys high sensitivity to an initial axial load and also withstands large compressive axial strain before buckling. As a result, they could be an ideal candidate for nanoscale strain/stress sensors applications.

\section{References}

1. S. Iijima, Nature 354,56 (1991).

2. R. F. Gibson, E. O. Ayorinde, and Y. F. Wen, Compos. Sci. Technol. 67, 1 (2006).

3. C. Y. Wang, Y. Y. Zhang, C. M. Wang, and V. B. C. Tan, J. Nanosci. Nanotechno. 7, 4221 (2007).

4. R. H. Baughman, A. A. Zakhidov, and W. A. de Heer, Science 297, 787 (2002).

5. K. T. Lau and D. Hui, Composites B 33, 263 (2002).

6. C. Y. Li and T. W. Chou, Phys. Rev. B 68, 073405 (2003).

7. C. Y. Li and T. W. Chou, Appl. Phys. Lett. 84, 121 (2004).

8. F. Scarpa and S. Adhikari, J. Non-Cryst. Sold. 354, 4151 (2008).

9. C. Y. Wang, C. Q. Ru, and A. Mioduchowskim, J. Appl. Mech. 71,622 (2004).

10. C. Y. Wang, C. Q. Ru, and A. Mioduchowski, J. Appl. Phys. 97, 024310 (2005).

11. C. Y. Wang, C. Q. Ru, and A. Mioduchowski, J. Appl. Phys. 97, 114323 (2005).

12. C. Y. Wang, C. Q. Ru, and A. Mioduchowski, Phys. Rev. B 72, 75414-1 (2005).

13. C. Y. Li and T. W. Chou, Nanotechnology 15, 1493 (2004).

14. Q. Zhao, J. R. Wood, and H. D. Wagner, Appl. Phys. Lett. 78, 1748 (2001).

15. R. Chowdhury, R. Adhikari, and J. Mitchell, Physica E: Lowdimensional Systems and Nanostructures (2009), to appear.

16. Y. Q. Zhang, G. R. Liu, and X. Han, Phys. Lett. A 340, 258 (2005).

17. H. Cai and X. Wang, Nanotechnology 17, 45 (2006).

18. X. Wang and H. Cao, Acta Mate. 54, 2067 (2006).

19. C. Sun and K. Liu, Solid State Communications 143, 202 (2007).

20. M. M. Selim, S. Abe, and K. Harigaya, Eur. Phys. J. B 69, 523 (2009).

21. C. Y. Wang and L. C. Zhang, Nanotechnology 19, 075705 (2008). 
22. C. Y. Wang and L. C. Zhang, Nanotechnology 19, 195704 (2008).

23. C. Y. Wang, C. Q. Ru, and A. Mioduchowski, J. Nnaosci. Nanotech. 3, 199 (2003).

24. C. Y. Wang, C. Q. Ru, and A. Mioduchowski, Int. J. Solid Struc. 40, 3893 (2003).

25. C. Y. Wang, C. Q. Ru, and A. Mioduchowski, J. Appl. Phys. 101, 014306 (2007).
26. F. M. Tong, C. Y. Wang, and S. Adhikari, J. Appl. Phys. 105, 094325 (2009).

27. V. N. Popov and V. E. Van Doren, Phys. Rev. B 61, 3078 (2000).

28. O. Dubay and G. Kresse, Phys. Rev. B 67, 035401 (2003).

29. S. S. Gupta, F. G. Bosco, and R. C. Batra, J. Appl. Phys. 106, 063527 (2009).

30. A. Sears and R. C. Batra, Phys. Rev. B 73, 085410 (2006).

Received: 28 September 2009. Accepted: 5 October 2009. 